DOI: $10.24850 /$ j-tyca-2020-01-06

Artículos

\title{
Caracterización de sequías hidrológicas en el río Cauca en su valle alto
}

\section{Characterization of hydrological drought in the Cauca river high valley}

Nathalia González-López ${ }^{1}$

Yesid Carvajal-Escobar ${ }^{2}$

${ }^{1}$ Universidad del Valle, Cali Colombia, nathalia.gonzalez.lopez@correounivalle.edu.co

${ }^{2}$ Universidad del Valle, Cali Colombia, yesid.carvajal@correounivalle.edu.co, ORCID: https://orcid.org/00000002-2014-4226

Autor para correspondencia: Yesid Carvajal-Escobar, yesid.carvajal@correounivalle.edu.co

\section{Resumen}

Las sequías son uno de los fenómenos naturales más complejos y pueden generar efectos devastadores para los sistemas sociales y 
ecológicos. Como consecuencia del cambio climático (CC); de la creciente presión de la demanda de agua causada por el crecimiento de la población; y la expansión de los sectores agrícola, energético e industrial, las sequías se han intensificado; por tanto, es necesario identificarlas y caracterizarlas. En este trabajo se calculó el índice de sequía de caudales (SDI) para cuatro estaciones ubicadas en el río Cauca en su valle alto, con el fin de detectar la ocurrencia e intensidad de las sequías hidrológicas. Los resultados mostraron que los eventos más intensos y de mayor magnitud ocurrieron en los años 1991-1992 y 2015-2016, durante los cuales se presentó el fenómeno El Niño en Colombia.

Palabras clave: caudales, El Niño, índice de sequía de caudales (SDI).

\section{Abstract}

Droughts are one of the most complex natural phenomena and can have devastating effects on social and ecological systems. As a consequence of the Climate Change (CC), the increasing pressure of water demand caused by population growth and the expansion of the agricultural, energy and industrial sectors have intensified, so it is necessary to identify and characterize droughts. In this work, the Streamflow Drought Index (SDI) was calculated for four stations located in the Cauca River in its high valley, with the purpose of detect the occurrence and intensity of hydrological droughts. The results showed that the most intense and major events occurred during the years 1991-1992 and 2015-2016, during which the El Niño phenomenon occurred in Colombia. 
Keywords: Streamflow, Streamflow Drought Index (SDI), El Niño.

Recibido: $13 / 08 / 2017$

Aceptado: 02/04/2019

\section{Introducción}

Desde la segunda mitad del siglo $X X$ se ha venido reportando en los ámbitos global, regional y local alteraciones espaciotemporales en las pautas de los fenómenos meteorológicos, como tormentas, granizadas y heladas, y en las variables climatológicas, como temperatura, humedad y precipitación, asociadas principalmente con la ocurrencia e intensidad de los fenómenos de la variabilidad climática (VC), cambio climático (CC) y El Niño-Oscilación del Sur (ENSO) (Forero, Hernández, \& Zafra, 2014; Pinilla, Rueda, Pinzón, \& Sánchez, 2012; Poveda \& Álvarez, 2012; Ulloa, 2014). El CC acelerado está generando efectos directos e indirectos relevantes y de suma complejidad, que se ven acentuados por la interacción con otros controladores del cambio global (cambios de uso del territorio, contaminación, intercambio biótico). 
Los recursos hídricos y los ecosistemas costeros, marinos y terrestres presentan los impactos más evidentes, los cuales, a su vez, afectan los medios de subsistencia y la salud de las poblaciones urbanas (Moreno, 2006; IPCC, 2014). El esquema de consecuencias en los recursos hídricos es diverso: ocurren alteraciones en el ciclo de agua, principalmente en el régimen de lluvias, mientras en ciertos sitios hay abundantes precipitaciones que, en intervalos cortos de tiempo, generan inundaciones; en otros hay periodos extendidos de sequías (Crossman et al., 2013; Cantú, 2014; Schewe et al., 2014); en ambos casos se ve afectada la disponibilidad y calidad del agua, y en consecuencia las actividades agrícolas, lo que puede poner en riesgo la subsistencia y seguridad alimentaria de las poblaciones, sobre todo en los países en desarrollo; situación que con el tiempo podría ser peor y aumentaría la desnutrición global si no se toman medidas al respecto (Wheeler \& Braun, 2013). Además, el aumento promedio previsto en la temperatura media anual de $2.5^{\circ} \mathrm{C}$ para el año 2050 y el incremento de $2.5 \%$ en la precipitación anual, probablemente provoque la degradación del suelo y pérdida de materia orgánica en las laderas de los Andes (Lau, Jarvis, \& Ramírez, 2013).

Las sequías son uno de los peligros naturales más perjudiciales y podrían resultar con efectos devastadores para los sistemas sociales y ecológicos; a diferencia de otros peligros naturales (inundaciones, deslizamientos), que por lo general se limitan a regiones relativamente pequeñas y se producen en intervalos temporales bien definidos, las sequías son tal vez las más costosas y menos previsibles, pues se desarrollan de forma gradual y sólo se identifican cuando están bien 
Tecnología y

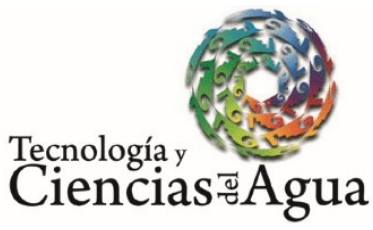

2020, Instituto Mexicano de Tecnología del Agua

Open Access bajo la licencia CC BY-NC-SA 4.0

(https://creativecommons.org/licenses/by-nc-sa/4.0/)

establecidas (Hao \& Aghakouchak, 2014; Hao \& Singh, 2015; Hong, Guo, Zhou, \& Xiong, 2015; Ravelo, Sanz, \& Douriet, 2014). Dada la gran variedad de sectores afectados, y su diversa distribución geográfica y temporal, es difícil tener una definición única y precisa de la sequía (Rajsekhar, Singh, \& Mishra, 2015), sin embargo, en términos generales, ésta ocurre cuando la disponibilidad de agua no logra satisfacer las demandas de las actividades humanas y del medio ambiente durante un periodo significativo y en un área extensa.

Considerando el numero víctimas y pérdidas en desastres de origen hidrometeorológico, la sostenibilidad social, económica y medioambiental puede y debe mejorarse con enfoques destinados a la gestión de riesgos de desastres y a la adaptación (Quintero-Angel, Carvajal-Escobar, \& Aldunce, 2012); por tanto, es indispensable realizar estudios destinados a la comprensión y caracterización de la sequía y a la modelización de sus componentes, sobre todo en aquellas zonas donde gran parte de la actividad económica depende del aprovechamiento del agua (Wagner, Rossi, Stahl, Bonal, \& Herault, 2012); para ello se pueden utilizar herramientas estadísticas que identifican la probabilidad de ocurrencia, duración e intensidad de un evento de sequía (Vicario et al., 2015).

Existen diferentes índices que sirven de insumos en los programas de prevención de desastres y generan bases científicas que pueden ser usadas en la gestión del riesgo y mitigación de sus impactos. Algunos requieren de cálculos complejos y de información robusta, dificultando su aplicación en zonas donde esta última es deficiente, pero existen 
otros que requieren sólo una variable, como la precipitación o el caudal, para ser calculados. Este trabajo busca identificar los extremos hidrológicos mínimos que se han presentado en el río Cauca en su valle alto, empleando el índice de sequía para caudales (SDI por sus siglas en inglés, Streamflow Drought Index); por medio de este índice se caracterizaron los eventos de sequía hidrológica, con base en su intensidad, duración y magnitud; información importante para la formulación de medidas de adaptación a dichos eventos, en una de la cuencas hidrografías más importantes de Colombia, en la cual el sector agropecuario se destaca como una de las principales actividades económicas y se puede ver gravemente afectado por la ocurrencia de eventos climáticos extremos.

\section{Metodología}

\section{Zona de estudio}

El río Cauca es la segunda fuente hídrica superficial más importante de Colombia, después del río Magdalena. Nace en el Macizo Andino 
Colombiano, entre las cordilleras Occidental y Central, en el departamento del Cauca, y desemboca en el río Magdalena; su elevación varía entre los 900 y 4000 msnm y cuenta con 39 afluentes, que influyen en el caudal promedio del río, llegando a aumentar hasta tres veces el caudal promedio desde la presa de Salvajina (unos 152 $\mathrm{m}^{3} / \mathrm{s}$ ) hasta su salida del departamento del Valle del Cauca $\left(568 \mathrm{~m}^{3} / \mathrm{s}\right.$ aprox.) (Enciso, Carvajal, \& Sandoval, 2016). En su recorrido el río Cauca pasa por más de 180 municipios.

La cuenca hidrográfica del río Cauca de unos 63300 km² presenta diversas actividades productivas, destacándose la industria azucarera, el cultivo de café, la generación de electricidad, y la explotación minera y agrícola (Pérez-Valbuena, Arrieta-Arrieta, \& Contreras-Anaya, 2015). Particularmente, la Cuenca Alta del Río Cauca, es de especial importancia estratégica, pues abarca casi todas las cadenas de producción priorizadas por el Ministerio de Agricultura de Colombia, así como una diversidad de sistemas agrícolas, que van desde pequeñas operaciones hasta granjas industriales; además es la base de gran parte de la agricultura técnica y de alto valor en el país (Peterson et al., 2012). La zona evaluada para el estudio fue la Cuenca Alta del Río Cauca, comprendida entre la estación La Balsa, ubicada a la entrada del Valle del Cauca, hasta la estación Anacaro ubicada a la salida del río del departamento; su ubicación se muestra en la Figura 1. 
Tecnología y

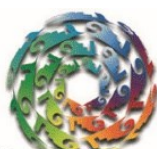

Ciencias $\stackrel{\Xi}{\triangleleft}$ gua
2020, Instituto Mexicano de Tecnología del Agua

Open Access bajo la licencia CC BY-NC-SA 4.0

(https://creativecommons.org/licenses/by-nc-sa/4.0/)

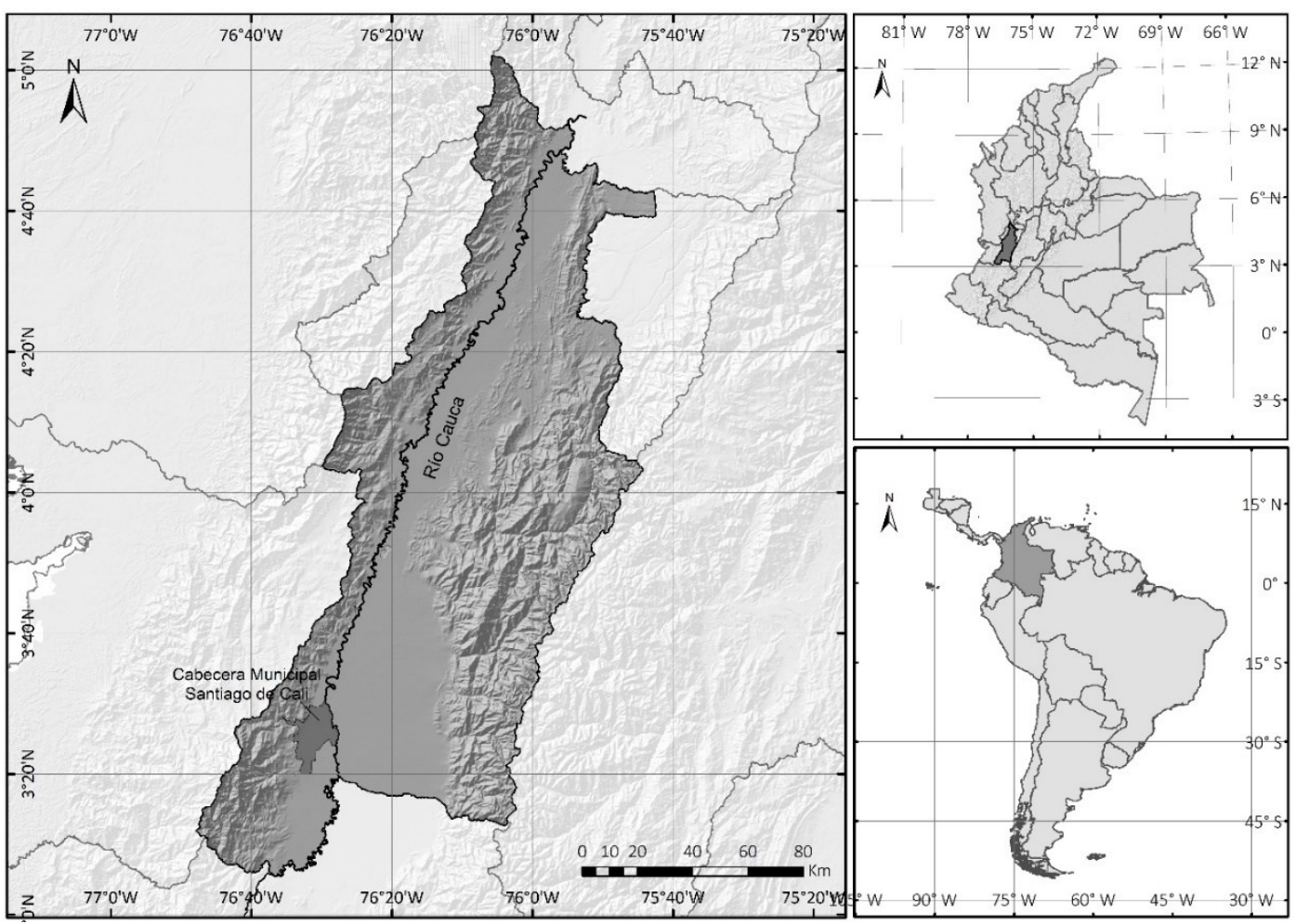

Figura 1. Ubicación de la zona de estudio.

\section{Método: SDI}

Las sequías se han intensificado como consecuencia del CC, de la creciente presión de la demanda de agua causada por el crecimiento de la población y de la expansión de los sectores agrícola, energético e industrial, entre otros. Lo anterior hace que sea indispensable 
Tecnología y

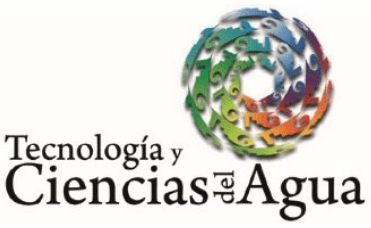

2020, Instituto Mexicano de Tecnología del Agua

Open Access bajo la licencia CC BY-NC-SA 4.0

(https://creativecommons.org/licenses/by-nc-sa/4.0/)

monitorear las condiciones de sequía; una de las formas de hacerlo es por medio de índices, los cuales proporcionan un método cuantitativo para determinar el inicio y fin de un evento de sequía (Tabari, Nikbakht, \& Hosseinzadeh-Talaee, 2013), así como la intensidad, magnitud y frecuencia. Existen diferentes índices para analizar la sequía hidrológica, dentro de los que destacan: el índice de sequía hidrológica de Palmer (PDSI), el índice de suministro de agua superficial (SWSI), el índice de deficiencia de escurrimiento regional (RDI), el índice de escurrimiento estandarizado (SRI) y el índice de sequía de caudales (SDI) (Hao \& Aghakouchak, 2014; Hao \& Singh, 2015; Hong et al., 2015; Rajsekhar et al., 2015; Ravelo et al., 2014; Tabari et al., 2013). En general, los índices para caracterizar la sequía hidrológica requieren de bastante información y de cálculos intensivos; sin embargo, el SDI es uno de los índices más sencillos y eficaces que se ha propuesto de manera reciente (Esquivel-Arriaga, Bueno, Sánchez-Cohen, Velásquez-Valle, \& EsquivelArriaga, 2014), al requerir sólo valores de caudal y su metodología; características y ventajas son análogas al índice de precipitación estandarizada (SPI), uno de los índices más usados en el orbe para caracterizar sequías meteorológicas.

Si bien la sequía hidrológica se define como una disminución significativa de la disponibilidad del agua en todas las formas que aparece en el ciclo hidrológico, el caudal permite conocer el comportamiento de los recursos hídricos superficiales $y$, desde el punto de vista de cantidad de agua, es la variable más importante (Nalbantis \& Tsakiris, 2009); además, incluye de modo indirecto otras variables climáticas, como precipitación y evapotranspiración, por tanto 
Teçnología y

\section{Ciencias ฐAgua}

2020, Instituto Mexicano de Tecnología del Agua

Open Access bajo la licencia CC BY-NC-SA 4.0

(https://creativecommons.org/licenses/by-nc-sa/4.0/)

proporciona información holística; además, casi todos los efectos de la sequía están relacionados con la sequía hídrica del suelo o la sequía hidrológica, ya que tanto el ecosistema como la sociedad dependen del agua de los reservorios (suelos, acuíferos, lagos y ríos). Por lo anterior, se decidió emplear el SDI, que es un índice integrador por usar información de caudales, lo que, además, es especialmente útil en zonas con poca información. De acuerdo con la metodología de Nalbantis (2008), a partir series normalizadas de caudales acumulados es posible identificar un evento de sequía hidrológica con base en su desviación estándar. Para normalizar los datos, el autor empleó la distribución lognormal de dos parámetros y calculó el índice a a partir de la iError! No se encuentra el origen de la referencia.), cuyo resultado indica la intensidad de la sequía; los rangos se muestran en la Tabla 1.

$S D I_{i, k}=\frac{y_{i, k}-\bar{y}_{k}}{s_{y, k}}$

donde $y_{i, k}$ es el logaritmo natural del caudal acumulado con media $\left(\bar{y}_{k}\right)$ y desviación estándar $s_{y, k}$.

Tabla 1. Clasificación del SDI.

\begin{tabular}{|c|c|}
\hline SDI & Categoría \\
\hline Mayor que 0.0 & Sin sequía \\
\hline 0.0 que -1.0 & Sequía leve \\
\hline
\end{tabular}




\begin{tabular}{|c|c|}
\hline-1.0 que -1.5 & Sequía moderada \\
\hline-1.5 que -2.0 & Sequía severa \\
\hline Menor que -2.0 & Sequía extrema \\
\hline
\end{tabular}

Existen diferentes métodos para seleccionar la distribución más adecuada para la normalización; por lo regular incluyen métodos gráficos y estadísticos. Para este trabajo se realizaron gráficas $Q-Q$ y se aplicaron las pruebas de Kolmogorov-Sminov (KS) y Anderson-Darling (AD) para seleccionar la distribución adecuada para normalizar las series de caudal acumulado en 6 y 12 meses. Se seleccionaron estas agrupaciones, ya que al igual que el SPI, las series mensuales y trimestrales presentan alta sensibilidad, según Núñez-López, MuñozRobles, Reyes-Gómez, Velasco-Velasco y Gadsden-Esparza (2007), cada registro mensual ejerce un efecto significativo en el total acumulado, dado que representa $100 \%$ para el acumulado mensual $y$, en promedio, $33.3 \%$ del trimestral; mientras que en series semestrales, el índice se estabiliza y define con mayor claridad el déficit hídrico, pues cada registro mensual representa sólo $16.6 \%$ para el acumulado en seis meses y $8.33 \%$ en 12 meses. Además, en regiones donde normalmente se dan condiciones secas durante algún periodo concreto, como es el caso de la zona de estudio, emplear el índice para 1 o 3 meses podría conducir a malinterpretaciones (OMM, 2012). 


\section{Resultados}

La hidrometeorología de la cuenca alta del río Cauca está fuertemente influenciada por el fenómeno ENSO. Presenta un régimen bimodal de lluvias, como resultado del doble paso de la zona de convergencia intertropical (ZCIT). Los dos periodos con mayores precipitaciones son marzo-abril-mayo y septiembre-octubre-noviembre, y los dos con menores precipitaciones son diciembre-enero-febrero y junio-julioagosto. Como respuesta a la bimodalidad de la precipitación, el caudal del río Cauca en su valle alto también tiene periodos de mayor y menor caudal; el trimestre julio, agosto y septiembre se caracteriza por ser el más seco, mientras noviembre, diciembre y enero presenta los caudales promedios más altos. 
Tecnología y
2020, Instituto Mexicano de Tecnología del Agua

Open Access bajo la licencia CC BY-NC-SA 4.0

(https://creativecommons.org/licenses/by-nc-sa/4.0/)

Para este estudio se consideraron cuatro estaciones: La Balsa, Juanchito, La Victoria y Anacaro, como se puede ver en la

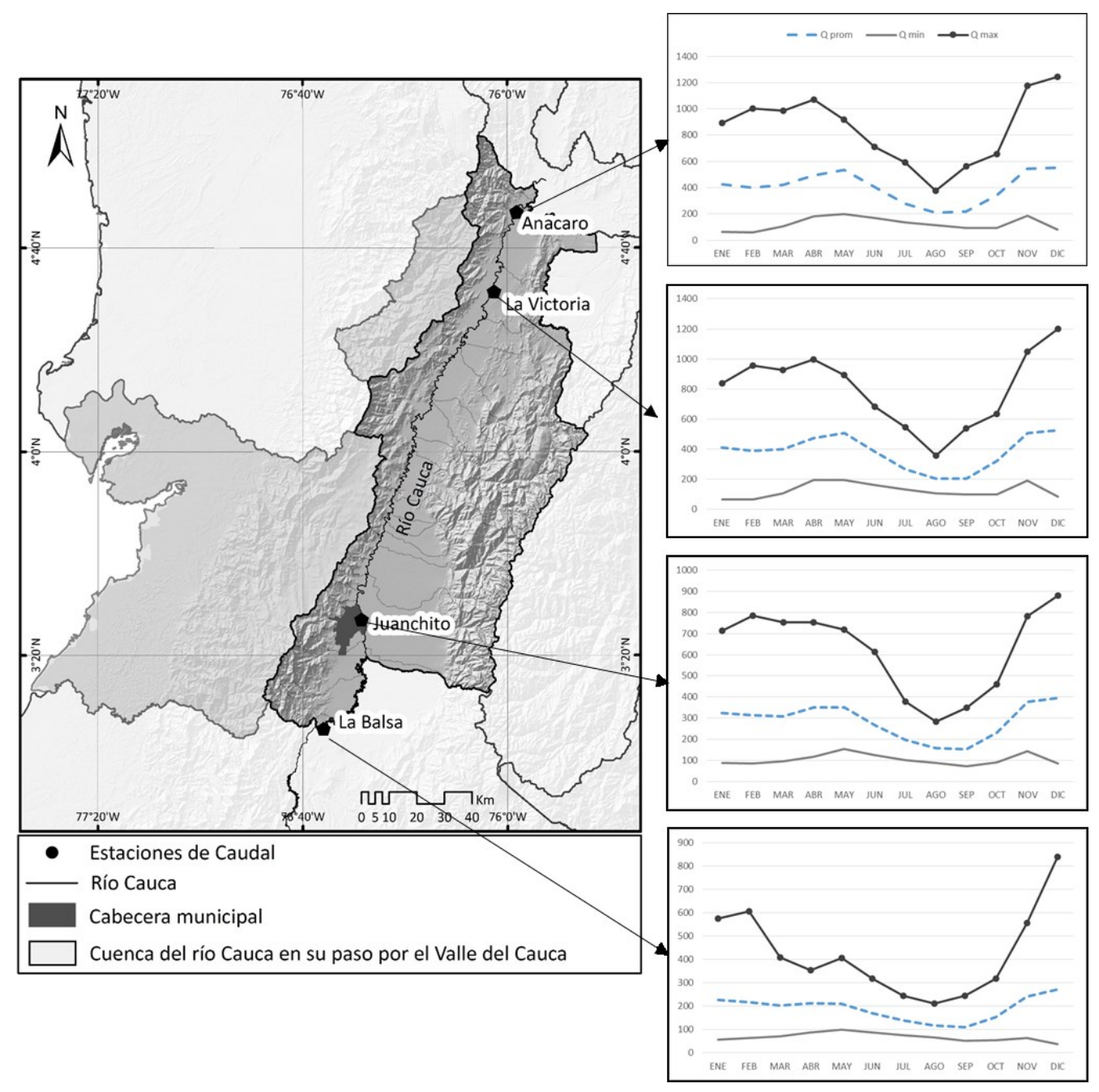

Figura 22, en la cual también se muestra el comportamiento de los caudales medios mensual multianuales. Los caudales registrados en la estación La Balsa, ubicada en la entrada del río al departamento, son menores en relación con los de salida, mientras que en Anacaro se han registrado valores máximos mensuales de hasta $1242 \mathrm{~m}^{3} / \mathrm{s}$; en La 
Tecnología y
2020, Instituto Mexicano de Tecnología del Agua

Open Access bajo la licencia CC BY-NC-SA 4.0

(https://creativecommons.org/licenses/by-nc-sa/4.0/)

Balsa, el máximo fue de $838 \mathrm{~m}^{3} / \mathrm{s}$, resultado que evidencia la influencia de los aportes de agua de los efluentes sobre el río.

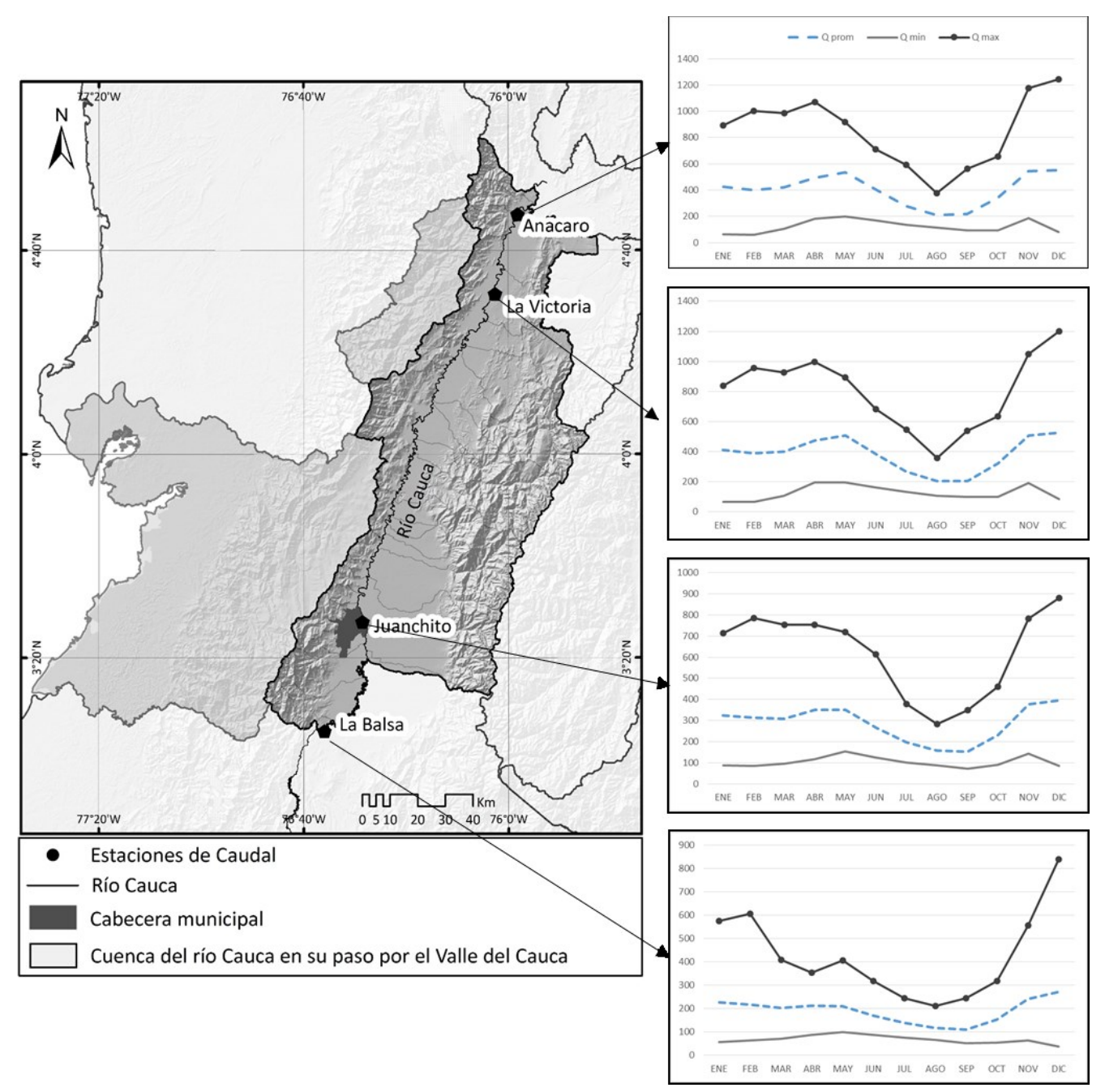

Figura 2. Estaciones hidrológicas de estudio.

Para la caracterización de los eventos de sequía hidrológica por medio del SDI, al inicio se llevaron a cabo pruebas estadísticas, con el fin de seleccionar la distribución adecuada para normalizar las series de 
caudal, ya que éstas no presentaban una distribución normal. Con las gráficas $Q-Q$ se comparó la distribución de los datos con las distribuciones normal, log-normal, exponencial y gamma; los resultados obtenidos para la serie de caudal acumulado en seis meses de la estación Anacaro se muestran en la Figura 3, a partir de cuyos datos se identificó que la distribución que presenta el mejor ajuste es log-normal; resultados similares se obtuvieron en las otras tres estaciones. Adicional al ajuste gráfico, se realizaron las pruebas KS y DA. A manera de ejemplo, se presentan en la Tabla 2 los resultados obtenidos para las series de caudales acumuladas en seis meses registrados en la estación Anacaro; los resultados indicaron para todos los casos que la distribución adecuada para normalizar los datos es log-normal. 
Tecnología y

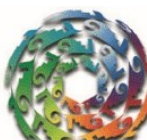

Ciencias $\approx$ Agua
2020, Instituto Mexicano de Tecnología del Agua

Open Access bajo la licencia CC BY-NC-SA 4.0

(https://creativecommons.org/licenses/by-nc-sa/4.0/)
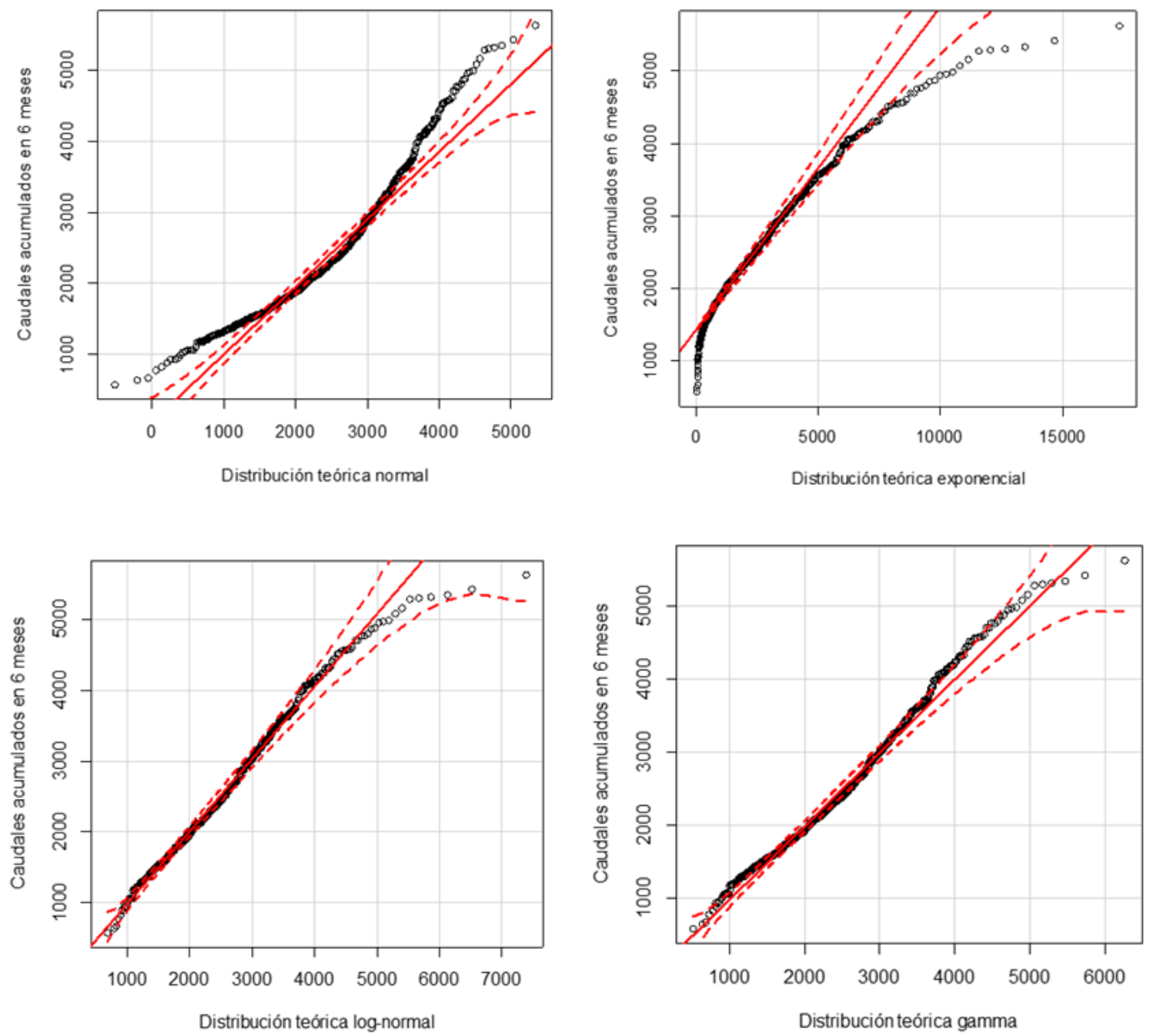

Figura 3. Gráficas $Q-Q$ para caudales acumulados en seis meses de la estación Anacaro en $\mathrm{m}^{3} / \mathrm{s}$.

Tabla 2. Resultados de las pruebas KS y DA para la estación Anacaro.

\begin{tabular}{|c|c|c|c|c|c|}
\hline & & Normal & Log-Normal & Exponencial & Gamma \\
\hline y đ Parámetros & Mean & 2417.2340 & 7.7207 & - & - \\
\hline
\end{tabular}




\begin{tabular}{|l|l|l|c|c|c|c|}
\hline & sd & 923.5320 & 0.3743 & - & - \\
\cline { 3 - 7 } & Rate & - & - & $4.14 \mathrm{E}-04$ & 0.0030 \\
\cline { 3 - 7 } & Shape & - & - & - & 7.3403 \\
\cline { 3 - 7 } & $\begin{array}{l}\text { Kolmogorov- } \\
\text { Smirnov }\end{array}$ & $\mathrm{D}$ & 0.0988 & 0.0255 & 0.3644 & 0.0506 \\
\cline { 2 - 6 } & P-Value & 0.00001 & 0.7892 & 0.0000 & 0.0702 \\
\cline { 2 - 6 } & $\begin{array}{l}\text { Anderson- } \\
\text { Darling }\end{array}$ & An & 12.0100 & 0.8140 & 123.3600 & 2.8220 \\
\cline { 2 - 6 } & P-Value & 0.0000 & 0.4710 & 0.0000 & 0.0337 \\
\hline
\end{tabular}

Nota: el $p$-value $>0.05$ señala que los datos observados se ajustan a la distribución evaluada, con un nivel de confianza de $95 \%$.

Los datos de caudal acumulados en 6 y 12 meses se normalizaron empleando la función de distribución Log-normal, por tanto, se siguió la metodología usada por Nalbantis (2008) y se calculó el índice por medio de la Ecuación (1). Independientemente del sistema analizado (hidrológico, agrícola o ecológico), se ha comprobado una mayor capacidad de los índices de sequía que pueden calcularse a diferentes escalas de tiempo (SPEI, SPI, SDI), pues se correlacionan mejor con la variabilidad temporal de las diferentes variables (Vicente-Serrano et al., 2012); el SDI permitió identificar por medio de desviaciones estándar, los periodos en los cuales los caudales acumulados en 6 y 12 meses estuvieron por debajo de la media. Las series se construyeron a partir de una suma móvil de los caudales mensuales; por tanto, para facilitar el manejo de la información, el mes al cual se hace alusión para describir cada evento corresponde al último mes de cada agrupación. En 
la Tabla 3 se presenta el periodo de registro de cada estación y el número de eventos calculados.

Tabla 3. Periodo de cada estación y número de eventos de sequía.

\begin{tabular}{|l|c|c|c|}
\hline \multirow{2}{*}{ Estación } & Periodo de registro & \multicolumn{2}{c|}{ Numero de eventos secos } \\
\cline { 3 - 4 } & & Semestrales & Anuales \\
\hline Anacaro & $1962-2016$ & 21 & 13 \\
\hline La Victoria & $1959-2016$ & 19 & 20 \\
\hline Juanchito & $1946-2016$ & 29 & 20 \\
\hline La Balsa & $1946-2016$ & 35 & 14 \\
\hline
\end{tabular}

En la Figura 4 se muestra el transcurso del SDI calculado con las series de caudal acumulado en seis meses, y en la Figura 5 en 12 meses de la estación Juanchito; al comparar las gráficas, se encontró que al aumentar la agrupación se reduce el número de eventos, pero éstos son de mayor duración. También se observó que existe una relación entre los eventos de sequías hidrológicas con los años en los cuales se presentó la fase cálida de fenómeno ENSO, El Niño, tales como 1958, 1977, 1992 y 2016. Los efectos del fenómeno ENSO sobre los caudales de los ríos en el país no son despreciables; en términos porcentuales, las principales afectaciones frente al fenómeno El Niño se presentan en la cuenca del río Magdalena-Cauca, con una reducción promedio de 26\% en los caudales, en la cuenca media del río Cauca de $38 \%$, en los ríos Sogamoso y Suárez hasta un 30\%; en el Sumapaz, las reducciones 
Tecnología y Ciencias $₫$ Agua
2020, Instituto Mexicano de Tecnología del Agua

Open Access bajo la licencia CC BY-NC-SA 4.0

(https://creativecommons.org/licenses/by-nc-sa/4.0/)

pueden llegar hasta un $40 \%$ y en el Urabá antioqueño entre 30 y $40 \%$ (García, Piñeros, Bernal, \& Ardila, 2012).

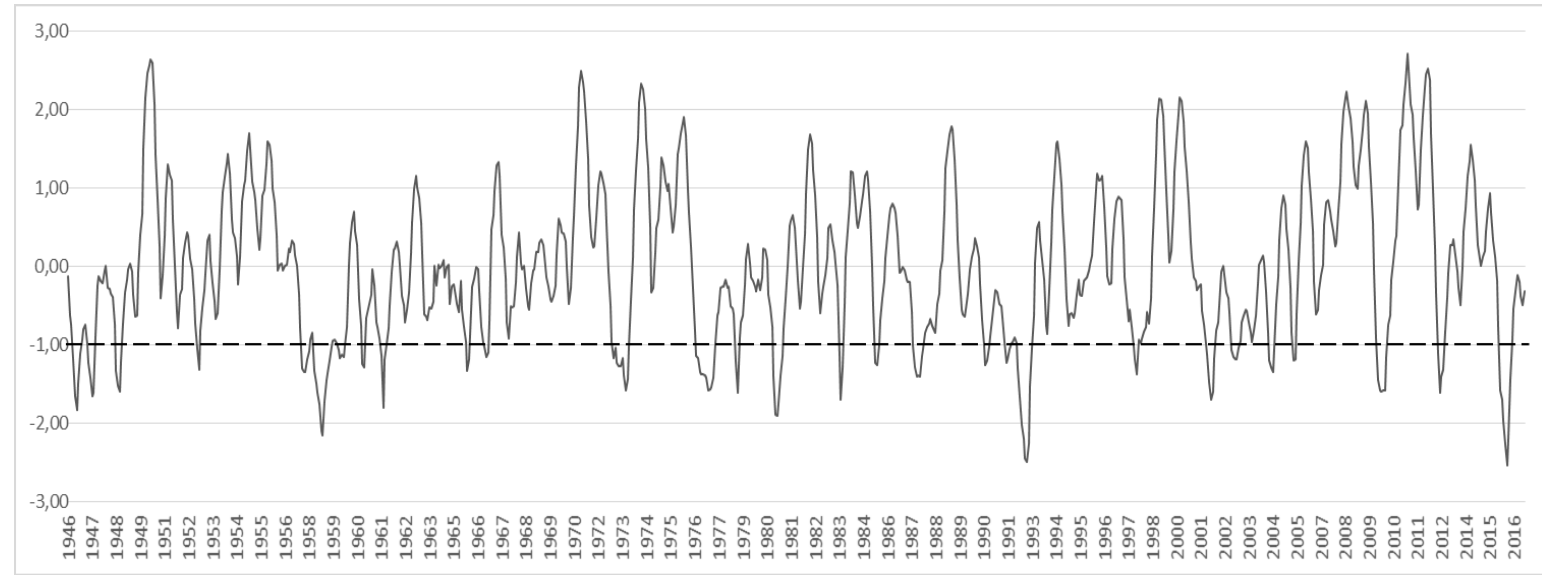

Figura 4. Transcurso del SDI $\mathrm{S}_{\mathrm{S}}$ en la estación Juanchito.

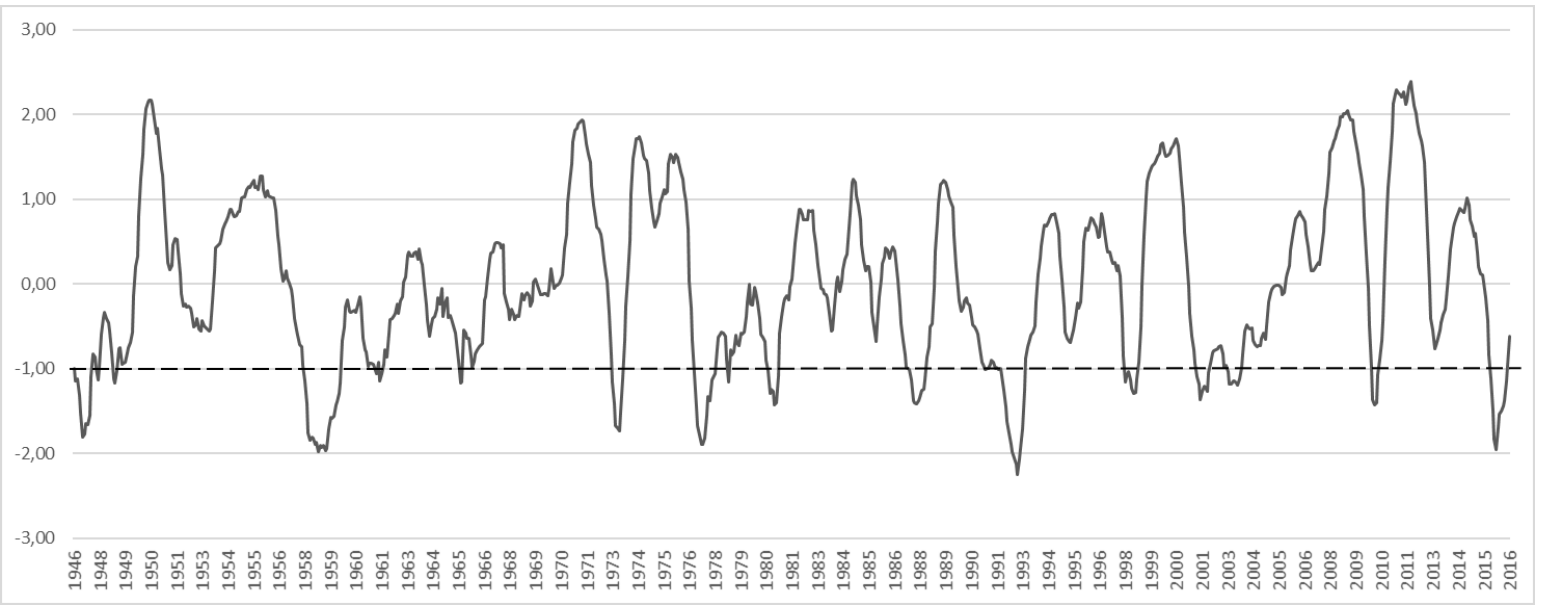

Figura 5. Transcurso del $\mathrm{SDI}_{\mathrm{A}}$ en la estación Juanchito. 
Teçnología y

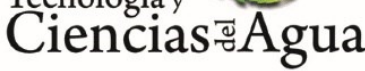

2020, Instituto Mexicano de Tecnología del Agua

Open Access bajo la licencia CC BY-NC-SA 4.0

(https://creativecommons.org/licenses/by-nc-sa/4.0/)

Los eventos de mayor duración están asociados con las mayores magnitudes, sin embargo, en ocasiones se presentan eventos de corta duración con grandes magnitudes. En la Tabla 4 se resumen los cinco eventos semestrales de mayor magnitud de cada estación, la fecha de inicio y finalización, duración y magnitud; y en la Tabla 5, para la agrupación anual. Las magnitudes más altas se presentaron en las estaciones La Victoria y Anacaro, ubicadas aguas abajo; resultado que además de reflejar posibles cambios en los factores climáticos, también pueden deberse a factores antrópicos, ocasionados por la toma de agua del río.

Tabla 4. Resumen de los cinco eventos de sequía de mayor magnitud para el SDI $\mathrm{S}$.

\begin{tabular}{|c|c|c|c|c|c|c|c|}
\hline \multirow[t]{2}{*}{ Estación } & \multirow[t]{2}{*}{ Evento } & \multicolumn{2}{|c|}{$\begin{array}{l}\text { Fecha de } \\
\text { inicio }\end{array}$} & \multicolumn{2}{|c|}{$\begin{array}{c}\text { Fecha de } \\
\text { finalización }\end{array}$} & \multirow[t]{2}{*}{ Duración } & \multirow[t]{2}{*}{ Magnitud } \\
\hline & & Año & Mes & Año & Mes & & \\
\hline \multirow{5}{*}{ Anacaro } & 1 & 2015 & 9 & 2016 & 12 & 16 & 33.61 \\
\hline & 2 & 1992 & 5 & 1993 & 2 & 10 & 17.76 \\
\hline & 3 & 2009 & 11 & 2010 & 5 & 7 & 12.60 \\
\hline & 4 & 1977 & 1 & 1977 & 9 & 9 & 12.31 \\
\hline & 5 & 1980 & 8 & 1981 & 1 & 6 & 8.58 \\
\hline \multirow{4}{*}{$\begin{array}{c}\text { La } \\
\text { Victoria }\end{array}$} & 1 & 2015 & 9 & 2016 & 12 & 16 & 23.13 \\
\hline & 2 & 1992 & 6 & 1993 & 2 & 9 & 12.68 \\
\hline & 3 & 1977 & 1 & 1977 & 9 & 9 & 10.52 \\
\hline & 4 & 2009 & 11 & 2010 & 5 & 7 & 9.58 \\
\hline
\end{tabular}


Tecnología y

Ciencias $\sqrt{\simeq}$ Agua
2020, Instituto Mexicano de Tecnología del Agua

Open Access bajo la licencia CC BY-NC-SA 4.0

(https://creativecommons.org/licenses/by-nc-sa/4.0/)

\begin{tabular}{|c|c|c|c|c|c|c|c|}
\hline \multirow[t]{3}{*}{ Estación } & \multirow[t]{2}{*}{ Evento } & \multicolumn{2}{|c|}{$\begin{array}{l}\text { Fecha de } \\
\text { inicio }\end{array}$} & \multicolumn{2}{|c|}{$\begin{array}{c}\text { Fecha de } \\
\text { finalización }\end{array}$} & \multirow[t]{2}{*}{ Duración } & \multirow[t]{2}{*}{ Magnituc } \\
\hline & & Año & Mes & Año & Mes & & \\
\hline & 5 & 1980 & 8 & 1981 & 1 & 6 & 7.15 \\
\hline \multirow{5}{*}{ Juanchito } & 1 & 1958 & 5 & 1959 & 3 & 11 & 17.14 \\
\hline & 2 & 1992 & 6 & 1993 & 2 & 9 & 16.91 \\
\hline & 3 & 1976 & 11 & 1977 & 9 & 11 & 15.28 \\
\hline & 4 & 2015 & 10 & 2016 & 4 & 7 & 13.65 \\
\hline & 5 & 1972 & 11 & 1973 & 8 & 10 & 12.54 \\
\hline \multirow{5}{*}{ La Balsa } & 1 & 2015 & 9 & 2016 & 7 & 11 & 23.73 \\
\hline & 2 & 2001 & 5 & 2002 & 2 & 10 & 15.35 \\
\hline & 3 & 2009 & 10 & 2010 & 6 & 9 & 14.57 \\
\hline & 4 & 1992 & 7 & 1993 & 1 & 7 & 12.65 \\
\hline & 5 & 2002 & 7 & 2003 & 4 & 10 & 12.10 \\
\hline
\end{tabular}

Tabla 5. Resumen de los cinco eventos de sequía de mayor magnitud para el $\mathrm{SDI}_{\mathrm{A}}$.

\begin{tabular}{|c|c|c|c|c|c|c|c|}
\hline \multirow[t]{2}{*}{ Estación } & \multirow[t]{2}{*}{ Evento } & \multicolumn{2}{|c|}{$\begin{array}{c}\text { Fecha de } \\
\text { inicio }\end{array}$} & \multicolumn{2}{|c|}{$\begin{array}{c}\text { Fecha de } \\
\text { finalización }\end{array}$} & \multirow[t]{2}{*}{ Duración } & \multirow[t]{2}{*}{ Magnitud } \\
\hline & & Año & Mes & Año & Mes & & \\
\hline \multirow{5}{*}{ Anacaro } & 1 & 2015 & 10 & 2016 & 12 & 15 & 35.32 \\
\hline & 2 & 1992 & 3 & 1993 & 4 & 14 & 24.27 \\
\hline & 3 & 1977 & 4 & 1978 & 1 & 10 & 14.21 \\
\hline & 4 & 2003 & 1 & 2003 & 10 & 10 & 10.75 \\
\hline & 5 & 1987 & 10 & 1988 & 6 & 10 & 10.70 \\
\hline
\end{tabular}


Tecnología y

Ciencias $\cong$ Agua
2020, Instituto Mexicano de Tecnología del Agua

Open Access bajo la licencia CC BY-NC-SA 4.0

(https://creativecommons.org/licenses/by-nc-sa/4.0/)

\begin{tabular}{|c|c|c|c|c|c|c|c|}
\hline \multirow[t]{2}{*}{ Estación } & \multirow[t]{2}{*}{ Evento } & \multicolumn{2}{|c|}{$\begin{array}{l}\text { Fecha de } \\
\text { inicio }\end{array}$} & \multicolumn{2}{|c|}{$\begin{array}{c}\text { Fecha de } \\
\text { finalización }\end{array}$} & \multirow[t]{2}{*}{ Duración } & \multirow[t]{2}{*}{ Magnitud } \\
\hline & & Año & Mes & Año & Mes & & \\
\hline \multirow{5}{*}{ La Victoria } & 1 & 2015 & 9 & 2016 & 12 & 16 & 23.21 \\
\hline & 2 & 1992 & 6 & 1993 & 2 & 9 & 12.72 \\
\hline & 3 & 1977 & 1 & 1977 & 9 & 9 & 10.57 \\
\hline & 4 & 2009 & 11 & 2010 & 5 & 7 & 9.61 \\
\hline & 5 & 1980 & 8 & 1981 & 1 & 6 & 7.18 \\
\hline \multirow{5}{*}{ Juanchito } & 1 & 1958 & 3 & 1959 & 12 & 22 & 36.87 \\
\hline & 2 & 1992 & 3 & 1993 & 4 & 14 & 24.44 \\
\hline & 3 & 1977 & 3 & 1978 & 3 & 13 & 19.14 \\
\hline & 4 & 2016 & 1 & 2016 & 10 & 10 & 15.09 \\
\hline & 5 & 1947 & 1 & 1947 & 10 & 10 & 14.65 \\
\hline \multirow{5}{*}{ La Balsa } & 1 & 2001 & 7 & 2005 & 2 & 44 & 68.22 \\
\hline & 2 & 2015 & 10 & 2016 & 12 & 15 & 30.58 \\
\hline & 3 & 1958 & 5 & 1959 & 9 & 17 & 20.12 \\
\hline & 4 & 1992 & 6 & 1993 & 4 & 11 & 16.18 \\
\hline & 5 & 2010 & 2 & 2010 & 10 & 9 & 14.41 \\
\hline
\end{tabular}

La sequía hidrológica está determinada por la propagación de la sequía meteorológica a través del ciclo hidrológico terrestre y, por lo 
tanto, está influenciada por las propiedades de este último (Van Loon \& Laaha, 2015). Con base en los resultados, las sequías hidrológicas de mayor magnitud se presentaron durante 2015 y comienzos de 2016, cuando ocurrió uno de los eventos más fuertes de El Niño en la historia. De acuerdo con el periódico (El Tiempo, 2016), este fenómeno desencadenó en promedio 14 incendios durante los 15 meses que duró; Colombia perdió por incendios forestales 188650 ha de bosques; las Iluvias se redujeron entre 30 y $40 \%$ y, en promedio, $80 \%$ de las zonas con influencia de El Niño tuvo un aumento de la temperatura hasta de $2.5{ }^{\circ} \mathrm{C}$. Esta sequía dejó los niveles históricos más bajos al río Magdalena y más de 200 municipios en calamidad por desabastecimiento de agua; la prevención y atención de emergencias le costó al país 1.6 billones de pesos. Lo anterior refleja la influencia del ENSO en el país, que durante la fase cálida, El Niño, genera principalmente una disminución de precipitación $y$, en consecuencia, de los caudales de los ríos, en la humedad del suelo y en la actividad vegetal (Rojo, 2011).

Si bien estos resultados son una aproximación a la caracterización de las sequías hidrológicas, es importante considerar que el SDI, al ser calculado con los valores de caudales (los cuales, a su vez, son estimados a partir de curvas de nivel-caudal) implica que puede tener un alto porcentaje de error. Las curvas de calibración para estimar los caudales tienen asociada una alta incertidumbre, y pueden ser simples o complejas, según el régimen de flujo y las características del cauce (Carvajal-Escobar, 2010). Asimismo, es importante considerar que durante los periodos secos sube la temperatura y con ello puede 
aumentar la evapotranspiración de los cultivos, incrementándose las necesidades de riego en algunos casos, ocasionando una mayor presión sobre los ríos; por consiguiente, sus niveles disminuyen al igual que los caudales no sólo por factores naturales sino también porque la demanda de agua de la sociedad es más demandantes. En ríos pequeños estos cambios afectan de modo significativo a los caudales y la incertidumbre en los cálculos es mayor; por ello se trabajó con los caudales del río Cauca, el cual tiene caudales mayores y más constantes en relación con otros ríos más pequeños.

\section{Conclusiones}

Los caudales de los ríos son uno de los elementos del ciclo hidrológico con mayores consecuencias sobre la vida de los seres humanos, por lo cual, identificar y caracterizar temporal y espacialmente los eventos hidrológicos extremos permite evaluar la disponibilidad hídrica regional y local, componente esencial en la planificación del agua. Si bien la sequía hidrológica se define como una disminución significativa de la disponibilidad del agua en todas las formas que aparece en el ciclo hidrológico, el caudal es la principal variable y permite conocer el comportamiento general de los recursos hídricos superficiales. Es por 
ello que el SDI constituye una herramienta importante para la caracterización de las sequías hidrológicas, sobre todo en zonas con poca información.

El río Cauca es la segunda fuente hídrica superficial más importante del país y a pesar de que normalmente se enfocan los estudios sobre este río a eventos máximos — pues a lo largo de los años ha registrado importantes crecientes que han causado inundaciones-, los resultados de este estudio indicaron que también se ha visto afectado por sequías hidrológicas, las cuales han ocasionado reducciones importantes en los caudales, principalmente en periodos en los que se ha presentado El Niño en el país, siendo los eventos de 1958, 1977, 1992 y 2016 los de mayores magnitudes y/o intensidades de sequía hidrológica.

En Colombia, la mayoría de los ríos de cuencas pequeñas no tiene registros $y$, en muchos casos, las estaciones están localizadas aguas abajo de las principales extracciones, por lo que extrapolar estos estudios a otros ríos puede dificultarse; sin embargo, se recomienda aplicarlo a otros ríos de importancia para el país.

\section{Agradecimientos}

Al Programa Jóvenes Investigadores e Innovadores de Colciencias 2015; al Grupo de Investigación en Ingeniería de Recursos Hídricos y Suelos; IREHISA; a la Universidad del Valle; a la CVC; al IDEAM, y a todas las entidades que suministraron la información y personas que contribuyeron de alguna forma en este trabajo. 


\section{Referencias}

Cantú, P. C. (2014). Cambio climático: sus repercusiones para la sustentabilidad. Sustentabilidad Ecológica, 17(67), 31-36.

Carvajal-Escobar, Y. (2010). Dimensiones para usar metodologías en la estimación de caudales ambientales en Colombia. Respuestas, 15(2), 34-46.

Crossman, J., Futter, M. N., Oni, S. K., Whitehead, P. G., Jin, L., Butterfield, D., Baulch, P. J., \& Dillon, P. J. (2013). Impacts of climate change on hydrology and water quality: Future proofing management strategies in the Lake Simcoe watershed, Canada. Journal of Great Lakes Research, 39(1), 19-32. Recuperado de https://doi.org/10.1016/j.jglr.2012.11.003

El Tiempo. (3 de junio, 2016). El Niño devastó por incendios área equivalente a tres veces Cali. Esta sequía dejó en los niveles históricos más bajos al río. Recuperado de http://www.eltiempo.com/estilo-de- vida/ciencia/efectos-delfenomeno-de-el- nino-en-colombia-2015-2016/16610226

Enciso, A., Carvajal, Y., \& Sandoval, M. C. (2016). Análisis hidrológico de las crecientes históricas del río Cauca en su valle alto. Revista Ingeniería y Competitividad, 18(1), 46-57.

Esquivel-Arriaga, G., Bueno, P., Sánchez-Cohen, I., Velásquez-Valle, M., \& Esquivel-Arriaga, O. (2014). Evaluación del índice de sequía de caudales en cuencas del norte-centro de México. Agrofaz, 14(2), 
Tecnología y

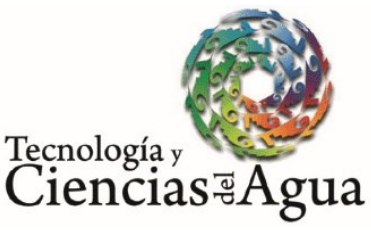

2020, Instituto Mexicano de Tecnología del Agua

Open Access bajo la licencia CC BY-NC-SA 4.0

(https://creativecommons.org/licenses/by-nc-sa/4.0/)

53-60.

Forero, E., Hernández, Y., \& Zafra, C. (2014). Percepción latinoamericana de cambio climático: metodologías, herramientas y estrategias de adaptación en comunidades locales. Una revisión. Revista UDCA Actualidad y Divulgación Científica, 17(1), 73-85.

García, M. C., Piñeros, A., Bernal, F. A., \& Ardila, E. (2012). Variabilidad climática, cambio climático y el recurso hídrico en Colombia. Revista de Ingeniería, 0(36), 60-64. Recuperado de https://doi.org/10.16924/riua.v0i36.136

Hao, Z., \& Aghakouchak, A. (2014). A nonparametric multivariate multiindex drought monitoring framework. Journal of Hydrometeorology, 15(1), 89-101. Recuperado de https://doi.org/10.1175/JHM-D-120160.1

Hao, Z., \& Singh, V. P. (2015). Drought characterization from a multivariate perspective: A review. Journal of Hydrology, 527, 668678. Recuperado de https://doi.org/10.1016/j.jhydrol.2015.05.031

Hong, X., Guo, S., Zhou, Y., \& Xiong, L. (2015). Uncertainties in assessing hydrological drought using streamflow drought index for the upper Yangtze River basin. Stochastic Environmental Research and Risk Assessment, 29(4), 1235-1247. Recuperado de https://doi.org/10.1007/s00477-014-0949-5

IPCC, Panel Intergubernamental del Cambio Climático. (2014). Cambio climático 2014. Impactos, adaptación y vulnerabilidad. Madrid, España: Panel Intergubernamental del Cambio Climático. 
Lau, C., Jarvis, A., \& Ramírez, J. (2013). Agricultura colombiana: adaptación al cambio climático. Cali, Colombia. CIAT Políticas en Síntesis no. 1. Cali, Colombia: Centro Internacional de Agricultura Tropical (CIAT)

Moreno, J. M. (2006). Evaluación preliminar de los impactos en España por efecto del cambio climático. Boletin $C F+S, 38 / 39,37-56$. Recuperado de https://doi.org/March 2006

Nalbantis, I. (2008). Evaluation of a Hydrological Drought Index. European Water, 2324, 67-77.

Nalbantis, I., \& Tsakiris, G. (March, 2009). Assessment of Hydrological Drought Revisited. Water Resources Management: An International Journal, Published for the European Water Resources Association (EWRA), Springer, European Water Resources Association (EWRA), 23(5), 881-897.

Núñez-López, D., Muñoz-Robles, C., Reyes-Gómez, V., Velasco-Velasco, I., \& Gadsden-Esparza, H. (2007). Caracterización de la sequía a diversas escalas de tiempo en Chihuahua, México. Agrociencia, $41(3), 253-262$.

OMM, Organización Meteorológica Mundial. (2012). Índice normalizado de precipitación Guía del usuario. Ginebra, Suiza: Organización Meteorológica Mundial.

Pérez-Valbuena, G. J., Arrieta-Arrieta, A. M., y Contreras-Anaya, J. G. (2015). Río Cauca: la geografía económica de su área de influencia. Economía Regional, (225), 17-52 
Tecnología y

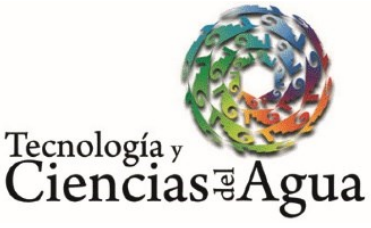

2020, Instituto Mexicano de Tecnología del Agua

Open Access bajo la licencia CC BY-NC-SA 4.0

(https://creativecommons.org/licenses/by-nc-sa/4.0/)

Peterson, C., Nowak, A., Jarvis, A., Navarrete, C., Figueroa, A., Riaño, N., \& Vargas, E. (2012). Un enfoque multi-dimensional de la cuenca alta del río Cauca de Colombia, con énfasis en la vulnerabilidad. Cenicafé, 63(2), 116-123.

Pinilla, M. C., Rueda, A., Pinzón, C., \& Sánchez, J. (2012). Percepciones sobre los fenómenos de variabilidad climática y cambio climático entre campesinos del centro de Santander, Colombia. Ambiente y Desarrollo, 16(31), 25-37.

Poveda, G., \& Álvarez, D. (2012). El colapso de la hipótesis de estacionariedad por cambio y variabilidad climática: implicaciones para el diseño hidrológico en ingeniería. Revista de Ingeniería, 36, 65-76.

Quintero-Angel, M., Carvajal-Escobar, Y., \& Aldunce, P. (2012). Adaptación a la variabilidad y el cambio climático: intersecciones con la gestión del riesgo. Revista Luna Azul, (34), 257-271. Recuperado de https://www.redalyc.org/articulo.oa?id=321727348015

Rajsekhar, D., Singh, V. P., \& Mishra, A. K. (2015). Multivariate drought index: An information theory based approach for integrated drought assessment. Journal of Hydrology, 526, 164-182. Recuperado de https://doi.org/10.1016/j.jhydrol.2014.11.031

Ravelo, A., Sanz, R., \& Douriet, J. C. (2014). Detección, evaluación y pronóstico de las sequías en la región del Organismo de Cuenca Pacífico Norte, México. Agriscientia, 31(1), 11-24.

Rojo, J. (2011). Desarrollo de un sistema experto para la predicción de caudales medios mensuales en Colombia. Recuperado de 
http://www.bdigital.unal.edu.co/5493/

Schewe, J., Heinke, J., Gerten, D., Haddeland, I., Arnell, N. W., Clark, D. B., Dankers, R., Eisner, S., Fekete, B. M., Colón-González, F. J., Gosling, S. N., Kim, H., Liu, X., Masaki, Y., Portmann, F. T., Satoh, T., Stacke, T., Tang, Q., Wada, Y., Wisser, D., Albrecht, T., Frieler, K., Piontek, F., Warszawski, L., \& Kabat, P. (2014). Multimodel assessment of water scarcity under climate change. Proceedings of the National Academy of Sciences of the United States of America, 111(9), 3245-50. Recuperado de https://doi.org/10.1073/pnas. 1222460110

Tabari, H., Nikbakht, J., \& Hosseinzadeh-Talaee, P. (2013). Hydrological drought assessment in Northwestern Iran Based on Streamflow Drought Index (SDI). Water Resources Management, 27(1), 137151. Recuperado de https://doi.org/10.1007/s11269-012-0173-3

Ulloa, A. (2014). Dimensiones culturales del clima: indicadores y predicciones entre pobladores locales en Colombia. Batey: Revista Cubana de Antropología Sociocultural, 6, 17-33.

Van Loon, A. F., \& Laaha, G. (2015). Hydrological drought severity explained by climate and catchment characteristics. Journal of Hydrology, 526, 3-14.

Vicario, L., García, C. M., Teich, I., Bertoni, J. C., Ravelo, A., \& Rofríguez, A. (2015). Caracterización de las sequías meteorológicas en la región central de la Argentina. Tecnología y ciencias del agua, 6(1), 153-165.

Vicente-Serrano, S., Beguería, S., Lorenzo-Lacruz, J., Camarero, J., 
López-Moreno, J., Azorín-Molina, C., Revuelto, J., Morán-Tejeda, E., \& Sánchez-Lorenzo, A. (2012). Análisis comparativo de diferentes índices de sequía para aplicaciones ecológicas, agrícolas e hidrológicas. In Asociación Española de Climatología (ed.). 80 Congreso Internacional de la Asociación Española de Climatología (pp. 679-689). Salamanca.

Wagner, F., Rossi, V., Stahl, C., Bonal, D., \& Hérault, B. (2012). Water availability is the main climate driver of neotropical tree growth. Plos One, 7, DOI: 10.1371/journal.pone.0034074

Wheeler, T., \& Braun, J. V. (2013). Climate change impacts on global food security. Science, 341(2012), 508-513. Recuperado de http://science.sciencemag.org/content/341/6145/508 